\title{
QUANTIFICAÇÃO DE ALQUILBENZENO LINEAR SULFONATO EM ESTAÇÃO DE TRATAMENTO DE EFLUENTES E RIOS POR CROMATOGRAFIA LÍQUIDA DE ALTA EFICIÊNCIA E EXTRAÇÃO EM FASE SÓLIDA
}

\author{
Luiz G. Silva, Sávia Gavazza, Lourdinha Florencio e Mario T. Kato* \\ Departamento de Engenharia Civil, Universidade Federal de Pernambuco, Av. Acadêmico Hélio Ramos s/n, Cidade Universitária, \\ 50740-530 Recife - PE, Brasil
}

Recebido em 28/06/2016; aceito em 21/12/2016; publicado na web em 05/02/2017

\begin{abstract}
QUANTIFICATION OF LINEAR ALKYLBENZENE SULFONATE IN SEWAGE TREATMENT PLANTS AND RIVERS BY HIGH PERFORMANCE LIQUID CHROMATOGRAPHY AND SOLID PHASE EXTRACTION. The procedures to implement a chromatographic method for the quantification of linear alkyl benzene sulfonate (LAS) in the influent, effluent and sludge of a wastewater treatment plant (WWTP), and in river water and sediment, are described and evaluated. The columns octylsilane $\left(\mathrm{C}_{8}\right)$ and octadecylsilane $\left(\mathrm{C}_{18}\right)$ for high performance liquid chromatography were evaluated for separation of LAS homologues. The results showed that both columns separated the four homologues, but $\mathrm{C}_{18}$ was better for separation of the isomers of each homologue. This enabled to qualitatively evaluate the degradation of homologues in the WWTP, according to their respective isomers. Samples pretreatment by solid phase extraction (SPE) columns, strong anionic exchange (SAX) and $\mathrm{C}_{18}$, was also evaluated. SPE was necessary for water river samples, either to concentrate LAS or to remove interference in the matrix; however, for WWTP samples, SPE showed unnecessary. The recovery of LAS homologues, after the procedures for sample preparation, was 90 to $110 \%$ with an accuracy of 1 to 5\%. The WWTP was efficient in LAS removal from domestic sewage; however, the concentrations in water and sediments of the receiving body were higher than the limits established by legislation due to illegal discharges of untreated sewage.
\end{abstract}

Keywords: LAS homologues and isomers; anaerobic treatment; validation of chromatographic method; SPE pre-treatment.

\section{INTRODUÇÃO}

Alquilbenzeno linear sulfonato (LAS - linear alkylbenzene sulfonate) é o tensoativo aniônico mais utilizado como princípio ativo dos detergentes e sabões em pó, e por isso, o de maior produção (3 milhões de toneladas em 2000) e consumo mundial (18,2 milhões de toneladas em 2003). O produto comercial é uma mistura de homólogos e isômeros de posição com cadeias lineares contendo de 10 a 14 átomos de carbono e foi introduzido no mercado como substituto biodegradável do alquilbenzeno sulfonato (ABS - alkylbenzene sulfonate) ${ }^{1,2} \mathrm{O}$ LAS é produzido pela sulfonação do alquilbenzeno linear (LAB - linear alkylbenzene), obtido pela alquilação de Friedel Crafts. Neste processo são usados diferentes catalisadores, alterando o percentual dos isômeros e, consequentemente, a viscosidade, a solubilidade e a aplicação do produto final. Quando o ácido fluorídrico é utilizado como catalisador, o percentual dos isômeros em cada homólogo é quase equivalente (17 a 20\%); e quando o cloreto de alumínio é utilizado, são produzidos, majoritariamente, os isômeros 2-fenil (2Ø) (30\%), seguido do $3 \varnothing$ (20\%) e decrescendo gradualmente para os isômeros $4 \varnothing$ a $6 \varnothing(15$ a $16 \%) .{ }^{1,3}$

O maior consumo doméstico de LAS tem provocado o aumento das suas concentrações em esgotos sanitários $\left(1\right.$ a $\left.15 \mathrm{mg} \mathrm{L}^{-1}\right)$ que chegam nas estações de tratamento (ETE) ou nas águas de rio, bem como nos lodos de ETE ( $<1$ a $10 \mathrm{~g} \mathrm{~kg}^{-1}$ de matéria seca) e nos sedimentos de rio ( $<0,001$ a $0,005 \mathrm{~g} \mathrm{~kg}^{-1}$ de matéria seca). Concentrações de LAS maiores que $0,27 \mathrm{mg} \mathrm{L}^{-1} \mathrm{em}$ água ou $0,035 \mathrm{~g} \mathrm{~kg}^{-1}$ de matéria seca em lodos e sedimentos têm efeito tóxico nos ecossistemas aquático e terrestre. Por isso, há a necessidade de monitoramento das suas concentrações e estudos para avaliar os impactos e sua taxa de degradação no ambiente. Testes laboratoriais apontaram uma eficiência de degradação superior a 99\% em ambiente aeróbio, com

*e-mail: kato@ufpe.br tempo de meia vida de 12 a 18 h em água de rio e de 0,6 a 1,3 h em ETE com tratamento aeróbio por lodos ativados. Em ETE com tratamento anaeróbio, estudos apontaram para uma baixa taxa de biodegradação, destacando-se a sua precipitação como sais de cálcio e magnésio, adsorção ao lodo, sólidos suspensos e em sedimentos como os principais mecanismos da sua remoção..$^{2-10}$

No Brasil não existe um valor padrão para concentrações de LAS em efluentes tratados e águas superficiais, mas somente um valor padrão para a concentração de surfatantes aniônicos totais, determinados pelo método das substâncias ativas ao azul de metileno (MBAS - methylene blue active substance) ${ }^{11} \mathrm{O}$ Conselho Nacional do Meio Ambiente (CONAMA) determinou que as concentrações de surfatantes aniônicos totais, quantificadas pelo método MBAS, deveriam ser expressas em unidades de $\mathrm{mg} \mathrm{L}^{-1}$ de LAS, e não deveriam ser maiores que $0,5 \mathrm{mg} \mathrm{L}^{-1}$ de LAS em águas superficiais. ${ }^{12}$ A União Europeia e a Áustria definiram valores padrões para as concentrações de LAS em águas superfíciais de 0,35 e 0,27 mg L $\mathrm{mg}^{-1}$, respectivamente..$^{13,14}$ No entanto, como no Brasil, não há um limite máximo definido para a concentração de LAS em efluentes tratados.

A quantificação do LAS pode ser realizada pela cromatografia gasosa acoplada à espectrometria de massas $(\mathrm{CG} / \mathrm{EM})^{15-17}$ e pela cromatografia líquida de alta eficiência com fase reversa (CLAE-FR), com detectores de espectrometria de massas, fluorescência ou UV-vis. ${ }^{10,18-21}$

A extração do LAS de lodo e sedimento de rio pode ser realizada pela extração líquida pressurizada, extração assistida por micro-ondas, ultrassom ou Soxhlet. ${ }^{20,22,23}$ A concentração do LAS, quando abaixo do limite de quantificação do método cromatográfico, é realizada pela evaporação de uma alíquota adequada da amostra e o resíduo é redissolvido com um volume e proporção adequados de uma solução metanol:água. Ou ainda, pela extração em fase sólida (SPE - solid phase extraction), que também atua na remoção de interferentes que coeluem com os homólogos do LAS, utilizando as colunas de troca aniônica muito forte (SAX - strong anionic exchange) e fase reversa 
octadecilsilano $\left(\mathrm{C}_{18}\right)$, já que o LAS possui na sua estrutura uma porção não polar, a cadeia alquílica, e outra polar, o grupo fenilsulfonato, fazendo com que ele seja retido nas duas colunas. ${ }^{4,5,9,20,23-25}$

Dada a crescente preocupação sobre a presença de LAS em amostras ambientais, líquidas e sólidas, e levando-se em conta o maior uso de tratamento anaeróbio para esgotos sanitários, bem como os avanços nos estudos sobre a sua biodegradabilidade, é importante que a sua concentração seja quantificada por método analítico adequado. O objetivo do trabalho foi implantar uma metodologia empregando CLAE e SPE para a quantificação de LAS em afluente, efluente e lodo de ETE e em água e sedimento de rio.

\section{PARTE EXPERIMENTAL}

\section{Reagentes e materiais}

Foram utilizados acetonitrila e metanol grau cromatográfico da Merck; trietilamina, ácido etanoico, ácido clorídrico e hidróxido de sódio p.a. da Merck; água com resistividade de $18 \mathrm{M} \Omega \mathrm{cm}$, purificada em sistema Merck Millipore; as colunas de SPE utilizadas foram $\mathrm{C}_{18}$ e SAX AcuBond II de $500 \mathrm{mg}$ e $3 \mathrm{~mL}$ da Agilent. O padrão do surfatante aniônico comercial continha $25,5 \%$ de LAS (em massa) e a composiçãodos homólogos $\mathrm{C}_{10}, \mathrm{C}_{11}, \mathrm{C}_{12}$ e $\mathrm{C}_{13}$ era de $14,1 \%, 31,6 \%$, $30,2 \%$ e $23 \%$, respectivamente; e a dos isômeros 2 -fenil- $\mathrm{C}_{8}\left(2 \varnothing \mathrm{C}_{8}\right)$ e 2 -fenil- $\mathrm{C}_{16}\left(2 \varnothing \mathrm{C}_{16}\right)$ era de $81 \%$ e $91 \%$ (surrogates), respectivamente. Os padrões do LAS e surrogates foram obtidos com a CEPSA Química (San Roque, Espanha).

\section{Equipamentos}

Foi utilizado um cromatógrafo a líquido Agilent series 1100 com sistema de bomba quaternária, degaseificador da fase móvel, injetor automático com loop de $100 \mu \mathrm{L}$, compartimento termostatizado para coluna cromatográfica, detectores UV-vis e fluorescência. As colunas analíticas eram Agilent Zorbax eclipse, XDB, $\mathrm{C}_{8}(150$ x 4,6 mm e $5 \mu \mathrm{m})$ e LiChrospher $100 \mathrm{RP}-18$ ( 250 x 4,6 mm e $5 \mu \mathrm{m})$ da Merck.

\section{Procedimentos experimentais}

\section{Local das coletas}

As amostras de esgoto e lodo foram coletadas numa ETE composta por um reator anaeróbio de manta de lodo e fluxo ascendente (UASB - upflow anaerobic sludge blanket), seguido de uma lagoa de polimento para pós-tratamento (ETE Mangueira, Recife PE, coordenadas $08^{\circ} 05^{\prime} 41^{\prime \prime}$ de latitude sul e $34^{\circ} 55^{\prime} 31^{\prime \prime}$ de longitude oeste). As amostras de água e sedimento foram coletadas no corpo receptor do efluente final dessa ETE, o rio Jiquiá, com um leito de cerca de $9 \mathrm{~km}$ de extensão, da sua nascente até sua confluência com o rio Tejipió. $\mathrm{O}$ rio Jiquiá recebe tanto efluentes tratados, como não tratados de muitos outros bairros da bacia hidrográfica. ${ }^{26}$ As amostras da ETE foram coletadas no afluente (P1), efluente do UASB (P2), efluente da lagoa de polimento (P3) e lodo do reator. As amostras de água e sedimento no Jiquiá foram coletadas no ponto de encontro do canal do efluente da ETE com o leito do rio (J2), e em um ponto à montante (J1) e outro à jusante (J3).

\section{Método cromatográfico}

No uso das colunas $\mathrm{C}_{8}$ e $\mathrm{C}_{18}$, a fase móvel foi água com $5 \mathrm{mmol}$ $\mathrm{L}^{-1}$ de ácido etanoico e $5 \mathrm{mmol} \mathrm{L}^{-1}$ de trietilamina (solvente $\mathrm{A}$ ) e acetonitrila (solvente B); o fluxo da fase móvel foi de $1 \mathrm{~mL} \mathrm{~min}^{-1}$, o volume da amostra de $100 \mu \mathrm{L}$, a temperatura do compartimento termostatizado da coluna de $40{ }^{\circ} \mathrm{C}$, a detecção UV-Vis em $230 \mathrm{~nm}$ e a fluorescência em $230 \mathrm{~nm}$ (excitação) e $290 \mathrm{~nm}$ (emissão). Para a separação dos constituintes do LAS foi aplicado um gradiente na fase móvel; no método com a coluna $\mathrm{C}_{8}$, iniciou-se com $20 \%$ do solvente $\mathrm{B}$, atingiu-se $60 \%$ em $20 \mathrm{~min}$, retornou-se a $20 \%$ depois de $1 \mathrm{~min}$ e assim permaneceu por $6 \mathrm{~min}$; com a coluna $\mathrm{C}_{18}$ iniciou-se com $30 \%$ do solvente $\mathrm{B}$, atingiu-se $60 \%$ em $20 \mathrm{~min}$, permanecendo assim por $13 \mathrm{~min}$, retornou-se a $30 \%$ em $1 \mathrm{~min}$ e assim permaneceu por $6 \mathrm{~min}$.

\section{Extração do LAS do lodo da ETE e do sedimento do rio}

As amostras de lodo da ETE ( $1 \mathrm{~g})$ ou de sedimento do rio (10 g) foram secas a $105^{\circ} \mathrm{C}$ por $24 \mathrm{~h}$ e incorporadas com os surrogates para a concentração de $20 \mathrm{mg} \mathrm{L}^{-1}$. Em seguida, foram transferidas para o Soxhlet. Metanol $(150 \mathrm{~mL})$ foi utilizado para extrair o LAS e os surrogates por $8 \mathrm{~h}$, e o extrato obtido foi evaporado para $100 \mathrm{~mL}$.

Preparação das amostras da ETE e do rio sem utilização de SPE

Os extratos de lodo da ETE ou de sedimento do rio, em alíquotas de $1 \mathrm{~mL}$ e $10 \mathrm{~mL}$, respectivamente, foram evaporados a $60{ }^{\circ} \mathrm{C}$ e o resíduo redissolvido com $1 \mathrm{~mL}$ de metanol:água $(2: 3 \mathrm{v} / \mathrm{v})$. As amostras do afluente da ETE $(5 \mathrm{~mL})$, efluente do reator UASB $(10 \mathrm{~mL})$, efluente da lagoa de polimento $(10 \mathrm{~mL})$ ou da água do rio $(10 \mathrm{~mL})$ foram incorporados com os surrogates para a concentração de 20 $\mathrm{mg} \mathrm{L}^{-1}$; em seguida, a fase aquosa foi evaporada a $60{ }^{\circ} \mathrm{C}$ e o resíduo redissolvido com $1 \mathrm{~mL}$ de metanol:água $(2: 3 \mathrm{v} / \mathrm{v})$.

\section{Preparação das amostras da ETE e do rio com utilização de SPE}

O pré-tratamento das amostras com colunas SPE foi realizado com SAX e $\mathrm{C}_{18}$, individualmente e combinadas. Estas foram inicialmente ativadas, a SAX com $n$-hexano $(5 \mathrm{~mL})$, metanol $(5 \mathrm{~mL})$ e água $(5 \mathrm{~mL})$; e a $\mathrm{C}_{18}$ com metanol $(5 \mathrm{~mL})$ e água $(5 \mathrm{~mL})$. Em seguida, foram percolados os extratos do lodo $(1 \mathrm{~mL})$ ou sedimento do rio $(10 \mathrm{~mL})$, previamente diluídos com metanol:água $(7: 13 \mathrm{v} / \mathrm{v})$, para não comprometer a adsorção do LAS e surrogates nas colunas; ou as amostras do afluente da ETE (5 ou $10 \mathrm{~mL}$ ), efluente do reator UASB (10 ou $20 \mathrm{~mL}$ ), efluente da lagoa de polimento (10 ou 20 $\mathrm{mL})$ e água do rio $(10,50$ ou $100 \mathrm{~mL}$ ), previamente incorporadas com os surrogates para a concentração de $20 \mathrm{mg} \mathrm{L}^{-1}$. Em seguida, foi percolado metanol:água $(7: 13 \mathrm{v} / \mathrm{v})$ para limpeza das colunas $(5 \mathrm{~mL})$, e por fim os solventes para eluição do LAS e surrogates; com SAX, utilizou-se ácido clorídrico $4 \mathrm{~mol} \mathrm{~L}^{-1} \mathrm{em}$ metanol ( $5 \mathrm{~mL}$ ), e com $\mathrm{C}_{18}$, metanol $(5 \mathrm{~mL})$. Os eluatos tiveram toda fase orgânica evaporada a $60{ }^{\circ} \mathrm{C}$ e o resíduo redissolvido com $1 \mathrm{~mL}$ de metanol:água $(2: 3 \mathrm{v} / \mathrm{v})$. Quando as colunas de SPE foram combinadas, a SAX foi a primeira a ser utilizada, o seu eluato foi neutralizado com solução aquosa de hidróxido de sódio $4 \mathrm{~mol} \mathrm{~L}^{-1}$ e percolado na coluna $\mathrm{C}_{18}$. $\mathrm{O}$ fluxo médio de percolação dos solventes e amostras nas colunas foi de $10 \mathrm{~mL} \mathrm{~min}^{-1}$.

\section{Validação dos métodos analíticos}

Após os procedimentos analíticos estabelecidos, eles foram validados em termos de (i) adequação do modelo matemático linear (faixa linear de trabalho) para o intervalo de concentração das curvas analíticas e da sua significância estatística pela ANOVA; ${ }^{27}$ (ii) dos limites de detecção e quantificação a partir dos parâmetros das equações de regressão, ${ }^{28} \mathrm{e}$ (iii) do efeito matriz, ${ }^{29,30}$ para o método cromatográfico. Para os procedimentos de preparação das amostras com e sem utilização de SPE, foram verificadas a recuperação do LAS, os surrogates e a precisão dos métodos..$^{31,32}$

\section{RESULTADOS E DISCUSSÃO}

\section{Método cromatográfico}

Condições cromatográficas

As condições cromatográficas com as colunas analíticas $\mathrm{C}_{8} \mathrm{e}$ 
$\mathrm{C}_{18}$ resultaram na separação dos quatro homólogos do LAS (Figura 1). No entanto, no método com a coluna $\mathrm{C}_{8}$, apenas um isômero foi separado (Figura 1a), ao passo que com a coluna $\mathrm{C}_{18}$, um mínimo de quatro isômeros foram separados em todos os homólogos (Figura 1b). Se o único propósito fosse a análise quantitativa dos homólogos, o método com a coluna $\mathrm{C}_{8}$ seria melhor, pois o tempo de análise foi mais curto $(27 \mathrm{~min})$ do que o do método com a $\mathrm{C}_{18}$ (40 min). Entretanto, se fosse para identificar em produtos comerciais a utilização de LAS sintetizado com o catalisador HF (Figura 1b) ou $\mathrm{AlCl}_{3}$ (Figura 1c), como ocorreria a degradação dos homólogos, e consecutivamente do LAS, em termos dos isômeros (Figura 1d), o método com a coluna $\mathrm{C}_{18}$ seria melhor. Observa-se que a degradação dos homólogos do LAS iniciou-se nos isômeros com o grupo fenilsulfonato mais distante do final da cadeia linear (2Ø a $4 \varnothing)$ (Figura 1d).

\section{Validação do método cromatográfico}

A adequação do modelo linear ao intervalo de concentração das curvas analíticas foi verificada pela razão entre a média quadrática da falta de ajuste e a média quadrática do erro puro $\left(\mathrm{MQ}_{\mathrm{faj}} / \mathrm{MQ}_{\mathrm{ep}}\right)$, para qual os valores de $\mathrm{F}_{\text {calculado }}$ (Tabela 1 ) foram menores que $\mathrm{F}_{\text {tabelado }}(3,97)$ com 5 e 7 graus de liberdade e $p=0,05$, indicando não haver falta de ajuste do modelo no intervalo de concentração das curvas analíticas. A significância estatística das equações de regressão foi verificada pela razão entre a média quadrática da regressão e a média quadrática dos resíduos $\left(\mathrm{MQ}_{\mathrm{R}} / \mathrm{MQ}_{\mathrm{r}}\right)$, para qual os valores de $\mathrm{F}_{\text {calculado }}$ (Tabela 1) foram maiores que $\mathrm{F}_{\text {tabelado }}(4,75)$ com 5 e 7 graus de liberdade e $\mathrm{p}=$ 0,05 , indicando que o modelo linear foi significativo. O efeito matriz foi pouco pronunciado, com valores na faixa que corresponde à baixa interferência da matriz no sinal do detector $(-20 \%<$ efeito matriz < $20 \%$ ) (Tabela 1); portanto, não influenciando nos limites de detecção e quantificação do método, calculados a partir dos parâmetros das equações de regressão. ${ }^{25}$ Os resultados apresentados na Tabela 1 referem-se ao método com a coluna analítica $\mathrm{C}_{18}$, mas os do método com a $\mathrm{C}_{8}$ foram muito semelhantes.

\section{Preparação das amostras}

Métodos de preparação das amostras com e sem a utilização de SPE

A SPE foi utilizada nos casos em que interferentes coeluiram com os homólogos do LAS utilizando 5 e $10 \mathrm{~mL}$ da amostra, e nos casos de amostras com concentrações dos homólogos abaixo do limite de quantificação do método cromatográfico; nesse último foi necessária maior quantidade da amostra $(>10 \mathrm{~mL})$, o que levou à baixa exatidão e precisão e aumento dos interferentes na análise cromatográfica quando a SPE não foi utilizada. Na Figura 2 tem-se o cromatograma da amostra concentrada sem SPE (>10 mL) (Figura 2a) e da amostra concentrada com SPE utilizando apenas a coluna $\mathrm{C}_{18}(50 \mathrm{~mL}$ ) (Figura 2b); em ambos foram observados interferentes coeluindo com o homólogo $\mathrm{C}_{10}$. A remoção desses interferentes (Figura 2c) foi realizada com a coluna de SPE SAX, seguida da $\mathrm{C}_{18}$, e o cromatograma resultante foi similar ao do padrão do LAS (Figura 2d), garantindo a seletividade da metodologia analítica. A coluna SAX atuou na remoção dos interferentes não polares e $\mathrm{a}_{18}$, dos polares. Para as amostras da ETE, a coluna de SPE SAX ou $\mathrm{C}_{18}$ foi suficiente para remoção dos interferentes quando presentes, sendo utilizadas sequencialmente ( $\mathrm{SAX}$ seguida da $\mathrm{C}_{18}$ ) apenas nas amostras do rio.

\section{Recuperação e precisão}

\section{Validação dos métodos de preparação das amostras com e sem utilização de SPE}

A recuperação para os homólogos do LAS foi de 100 a $110 \%$ com precisão de 1 a $4 \%$ para as amostras do afluente da ETE, efluente do reator UASB, efluente da lagoa de polimento e água do
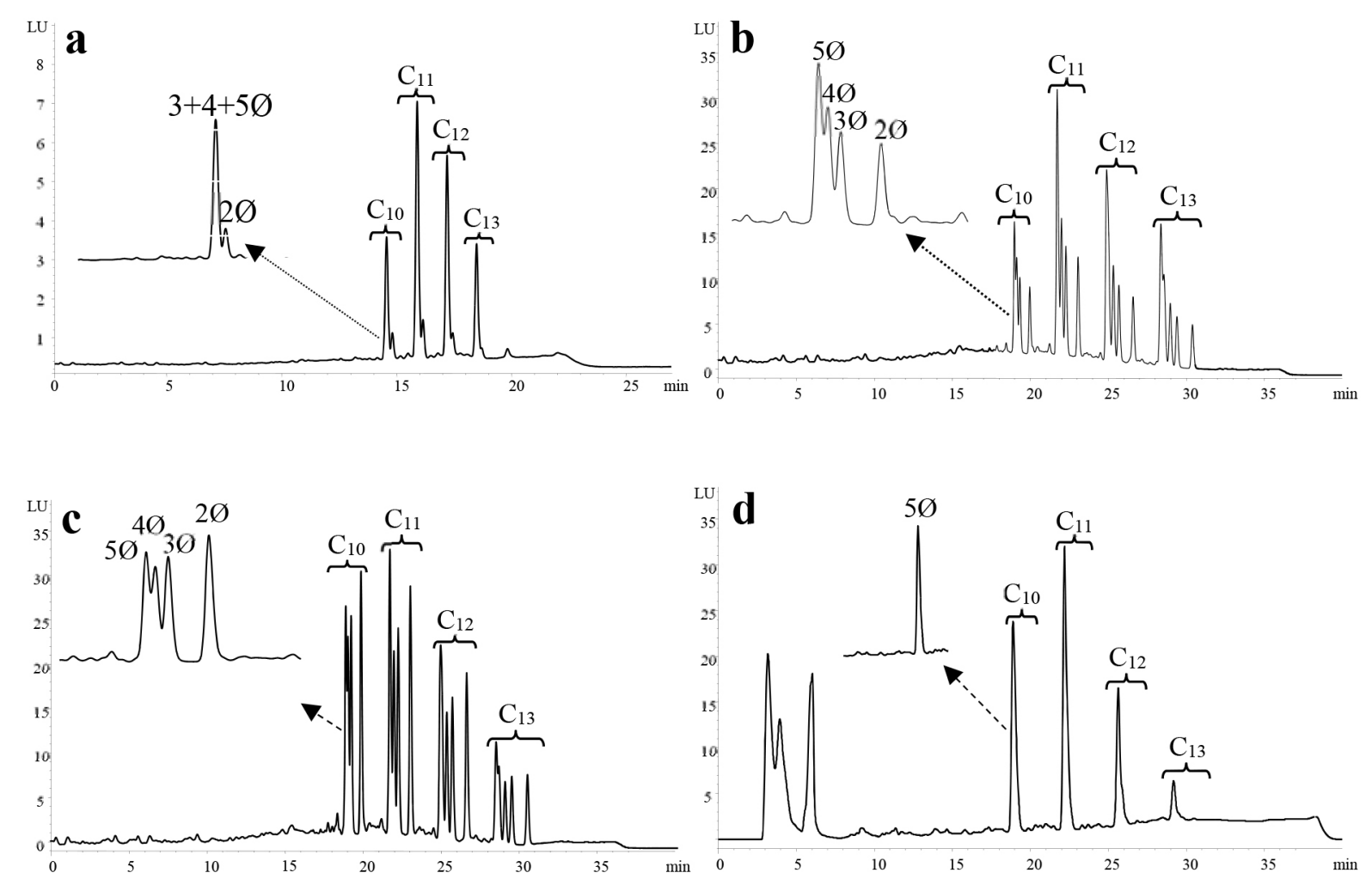

Figura 1. Cromatograma da separação dos homólogos e isômeros do LAS com a coluna $C_{8}(a)$ e $C_{18}(b)$ sintetizado com HF; da separação dos homólogos e isômeros do LAS com a coluna $C_{18}\left(\right.$ c) sintetizado com $\mathrm{AlCl}_{3}$ e degradação dos isômeros do LAS com a coluna $C_{18}(d)$ 
Tabela 1. Linearidade da curva analítica e limites de detecção e quantificação do método com coluna $\mathrm{C}_{18}$

\begin{tabular}{|c|c|c|c|c|}
\hline Parâmetros & $\mathrm{C}_{10}$ & $\mathrm{C}_{11}$ & $\mathrm{C}_{12}$ & $\mathrm{C}_{13}$ \\
\hline Inclinação da reta (a) & 122,4 & 122,2 & 112,0 & 104 \\
\hline Desvio padrão da inclinação da reta $\left(\mathrm{s}_{\mathrm{a}}\right)$ & 0,8 & 0,8 & 0,9 & 1 \\
\hline Intersecção da reta (b) & -2 & +8 & -3 & -3 \\
\hline Desvio padrão da intersecção da reta $\left(\mathrm{s}_{\mathrm{b}}\right)$ & 6 & 14 & 14 & 13 \\
\hline Coeficiente de correlação ajustado $\left(\mathrm{r}^{2}\right)$ & 0,9997 & 0,9997 & 0,9995 & 0,9990 \\
\hline $\mathrm{F}_{\text {calculado }}$ da $\mathrm{MQ}_{\mathrm{faj}} / \mathrm{MQ}_{\mathrm{ep}}$ & 0,79 & 1,15 & 0,86 & 0,50 \\
\hline $\mathrm{F}_{\text {calculado }}$ da $\mathrm{MQ}_{\mathrm{R}} / \mathrm{MQ}_{\mathrm{r}}$ & 24429 & 21886 & 16055 & 8974 \\
\hline Efeito matriz no sinal do detector $(\%)$ & $-10,4$ & $-8,9$ & $-7,3$ & $-9,6$ \\
\hline Faixa linearde trabalho $\left(\mathrm{mg} \mathrm{L}^{-1}\right)$ & $0,71-14,10$ & $1,58-31,60$ & $1,51-30,10$ & $1,15-23,00$ \\
\hline Limite de detecção $\left(\mathrm{mg} \mathrm{L}^{-1}\right)$ & 0,16 & 0,38 & 0,41 & 0,41 \\
\hline Limite de quantificação (mg L $\left.{ }^{-1}\right)$ & 0,49 & 1,15 & 1,25 & 1,25 \\
\hline
\end{tabular}
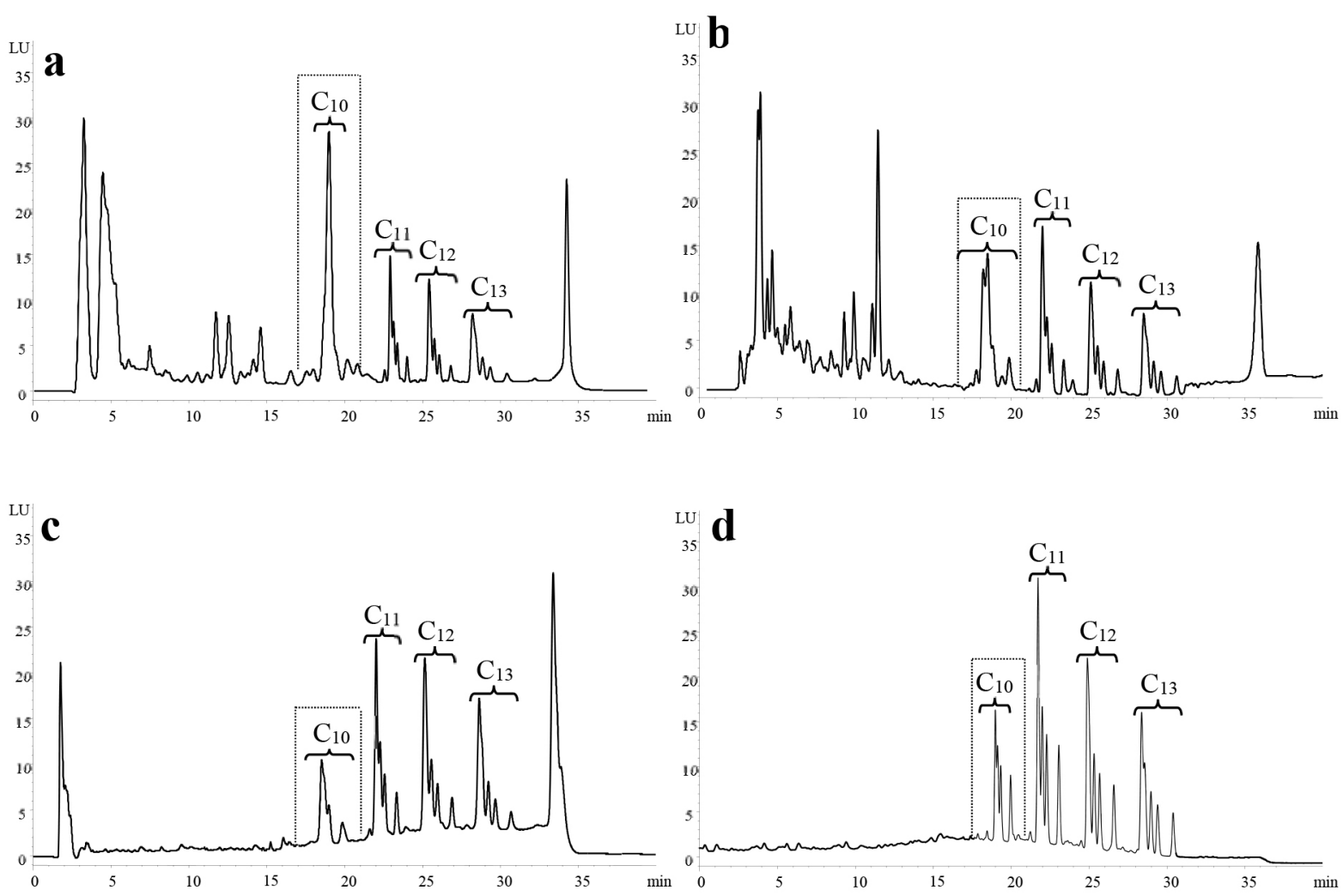

Figura 2. Cromatograma da separação dos homólogos e isômeros do LAS na amostras sem pré-tratamento com SPE (a), com pré-tratamento com coluna de SPE SAX (b), com pré-tratamento com colunas de SPE SAX seguida da $C_{18}($ c) e da solução padrão do LAS (d)

rio; e de 90 a $103 \%$ com precisão de 1 a $5 \%$ para amostras de lodo da ETE e de sedimento do rio (Tabela 2). Esses percentuais podem ser considerados satisfatórios e se encontram dentro da faixa de valores encontrada na literatura. ${ }^{19,28,33,34} \mathrm{~A}$ análise estatística (teste-t de Student independente) entre a recuperação dos homólogos do LAS e surrogates, nos casos sem e com utilização de SPE, revela que não houve diferenças significativas; ou seja, os valores do $t_{\text {calculado }}$ foram menores que $t_{\text {tabelado }}(1,860)$ com 8 graus de liberdade $\mathrm{e}=0,05$, com exceção dos casos em que interferentes coeluiram com os homólogos do LAS. Na Tabela 2 são apresentados os valores da recuperação pelos dois métodos utilizados na preparação das amostras. Aos valores da recuperação dos surrogates e homólogos do LAS das amostras do lodo da ETE e sedimento do rio, foram somadas também as variações nas concentrações devido ao processo de extração com o Soxhlet.
Tabela 2. Recuperação do LAS e precisão dos métodos analíticos $(\mathrm{n}=10)$

\begin{tabular}{lccccc}
\hline \multirow{2}{*}{ Composto } & \multicolumn{2}{c}{ Amostras aquosas $(\%)$} & & \multicolumn{2}{c}{ Amostras sólidas (\%) } \\
\cline { 2 - 3 } \cline { 6 - 6 } \cline { 5 - 6 } & Rio & ETE & & Rio & ETE \\
\hline Homólogo $C_{10}$ & $105 \pm 3$ & $103 \pm 2$ & & $102 \pm 2$ & $97 \pm 1$ \\
Homólogo $C_{11}$ & $106 \pm 1$ & $101 \pm 1$ & & $101 \pm 1$ & $95 \pm 1$ \\
Homólogo $C_{12}$ & $110 \pm 2$ & $101 \pm 3$ & & $102 \pm 1$ & $92 \pm 2$ \\
Homólogo $C_{13}$ & $109 \pm 2$ & $100 \pm 4$ & & $103 \pm 3$ & $90 \pm 5$ \\
Surrogate $2 \varnothing C_{8}$ & $100 \pm 1$ & $99 \pm 2$ & & $100 \pm 2$ & $98 \pm 2$ \\
Surrogate $2 \varnothing C_{16}$ & $98 \pm 2$ & $97 \pm 3$ & & $89 \pm 3$ & $86 \pm 6$ \\
\hline
\end{tabular}

O emprego dos surrogates possibilitou avaliar as interações dos homólogos do LAS com as matrizes e determinar os erros aleatórios 
devido a essas interações e os procedimentos de extração e preparação das amostras, principalmente para o lodo da ETE, para o qual foram obtidos os menores valores de recuperação (Tabela 2). O surrogate $2 \varnothing \mathrm{C}_{8}$ apresentou valores maiores que os do $2 \varnothing \mathrm{C}_{16}$, demonstrando que, quanto maior a cadeia linear do homólogo, maior sua adsorção e, consecutivamente, sua concentração no lodo da ETE e no sedimento do rio. Como observado na Figura 3a, a concentração dos homólogos do LAS em ordem crescente foi: $\mathrm{C}_{10}<\mathrm{C}_{11}<\mathrm{C}_{12}<\mathrm{C}_{13}$. O inverso ocorreu para o afluente da ETE, efluente do reator UASB e água do rio (Figura 3b).

A razão das áreas dos picos cromatográficos do surrogate $2 \varnothing \mathrm{C}_{8}$ e homólogos $\mathrm{C}_{10}$ e $\mathrm{C}_{11}$ gerou valores muito próximos, assim como do surrogate $2 \varnothing \mathrm{C}_{16}$ e os homólogos $\mathrm{C}_{12} \mathrm{e}_{13}$. Isso permitiu criar um fator para corrigir os desvios nas concentrações dos homólogos devido aos processos de preparação das amostras e análise cromatográfica, atuando também como um padrão interno.

\section{Aplicação dos métodos para quantificação do LAS em ETE e rio}

As concentrações de LAS no afluente da ETE (P1), efluente do reator UASB (P2) e efluente da lagoa de polimento (P3) diminuíram gradativamente de P1 para P3 (Figura 4a). A remoção total do LAS na ETE foi considerada satisfatória, sendo uma parcela removida no reator UASB e outra na lagoa de polimento (LP). Nesta unidade ocorreu uma maior eficiência de remoção do LAS (Figura 4b). No reator UASB, a remoção foi predominantemente por adsorção do LAS no lodo (Figura 4c). A massa de LAS adsorvida no lodo foi 461 a 1367 vezes maior que a massa dissolvida no afluente da ETE e efluente do reator UASB, respectivamente. Essa comparação foi realizada assumindo os mesmos volumes para as três amostras e as respectivas concentrações de LAS. Para o caso do lodo, foi determinada previamente a sua densidade $\left(\mathrm{d}=1,07 \pm 0,05 \mathrm{mg} \mathrm{L}^{-1}\right)$.

Na LP, os mecanismos atuantes na remoção do LAS foi a adsorção nos sólidos suspensos e degradação biológica. A sua degradação foi constatada qualitativamente a partir dos cromatogramas das análises do seu efluente com o método cromatográfico com a coluna analítica $\mathrm{C}_{18}$, como apresentado previamente na Figura 1d. Nesse, constatou-se a falta de sinal (picos) para os isômeros com o grupo fenilsulfonato mais distante da cadeia linear. Essa constatação também foi coerente com o ambiente da LP, o qual é predominantemente aeróbio, com níveis de oxigênio dissolvido de até $8 \mathrm{mg} \mathrm{L}^{-1}$, portanto, em condições nas quais a degradação biológica do LAS é largamente comprovada.

Na Figura 4d são apresentados os resultados das concentrações de LAS nas águas do rio Jiquiá; observa-se que, em geral, elas não diferiram entre os três pontos de coleta, exceto em setembro de 2009, quando ocorreram as maiores concentrações. Em todo o período de monitoramento e em todos os pontos de coleta, as concentrações de LAS foram superiores aos limites definidos por orgãos de controle ambiental para águas de rios. Nos sedimentos as suas concentrações foram acima de $0,035 \mathrm{~g} \mathrm{~kg}^{-1}$ de matéria seca (Figura 4e), que pode causar efeito tóxico para os organismos do ecossistema terreste. ${ }^{2,3}$ Essas concentrações maiores de LAS, tanto nas águas, como nos sedimentos, foram associadas ao aumento de moradias desordenadas às margens do rio e em seu entorno. Como nem todas as moradias existentes ou novas são atendidas por sistemas de tratamento de efluentes, o resultado foi um aumento gradual do lançamento de efluentes domésticos não tratados no rio Jiquiá ao longo dos anos. Caso isso não ocorresse, a única fonte de LAS para o rio, apesar de possuir baixíssima concentração, seria o efluente da ETE, o qual ao se misturar com as águas do rio ainda seria mais diluído; consequentemente, as concentrações do LAS no rio certamente se enquadrariam no valor padrão definido pela legislação ambiental.

$\mathrm{O}$ método com a coluna $\mathrm{C}_{18}$ também foi utilizado para avaliar a degradação do homólogo $\mathrm{C}_{10}$ e seus isômeros $\left(2 \varnothing \mathrm{C}_{10}, 3 \varnothing \mathrm{C}_{10}, 4 \varnothing \mathrm{C}_{10}\right.$ e $5 \varnothing \mathrm{C}_{10}$ ) (Figura 5a) por micro-organismos facultativos em reator em escala de laboratório. Ele mostrou que a degradação do homólogo $\mathrm{C}_{10}$ iniciou-se nos isômeros $2 \varnothing \mathrm{C}_{10}$ e $3 \varnothing \mathrm{C}_{10}$ (Figura 5b), seguido do $4 \varnothing \mathrm{C}_{10}$ (Figura $5 \mathrm{c}$ ). Esse resultado foi associado ao efeito do impedimento estérico, causado pelo grupo fenilsulfonato, o qual é menor nos isômeros em que ele está mais distante do final da cadeia linear, onde a degradação é iniciada. ${ }^{35,36}$ Essa constatação pode ser estendida aos demais homólogos, pois têm a mesma função química, diferindo apenas no número de átomos de carbonos na cadeia linear. Ainda, os isômeros com o grupo fenilsulfonato em uma mesma posição são mais facilmente degradados nos homólogos com cadeias lineares maiores, pois estão mais distantes do final da cadeia. Por exemplo, o isômero $2 \varnothing \mathrm{C}_{13}$ é degradado mais rapidamente que o $2 \varnothing \mathrm{C}_{12}$ e assim sucessivamente, logo, os homólogos com maior cadeia alquílica são também os mais facilmente degradados.

\section{CONCLUSÃO}

Os métodos cromatográficos com as colunas $\mathrm{C}_{8}$ e $\mathrm{C}_{18}$ foram excelentes na quantificação dos homólogos do LAS. No entanto, o método com a $\mathrm{C}_{18}$ foi melhor na separação dos isômeros e com isso foi possível avaliar qualitativamente a degradação dos homólogos do LAS em função da degradação dos seus respectivos isômeros.

A quantificação do LAS nas amostras do rio foi mais exata e precisa quando se utilizou as colunas de SPE SAX seguida da $C_{18}$, já para as amostras da ETE a SPE se mostrou desnecessária. A recuperação do LAS e surrogates após extração do lodo anaeróbio e sedimento de rio com Soxhlet, e os procedimentos para concentração do LAS utilizando ou não a SPE, foi alta, assim como a precisão dos métodos,
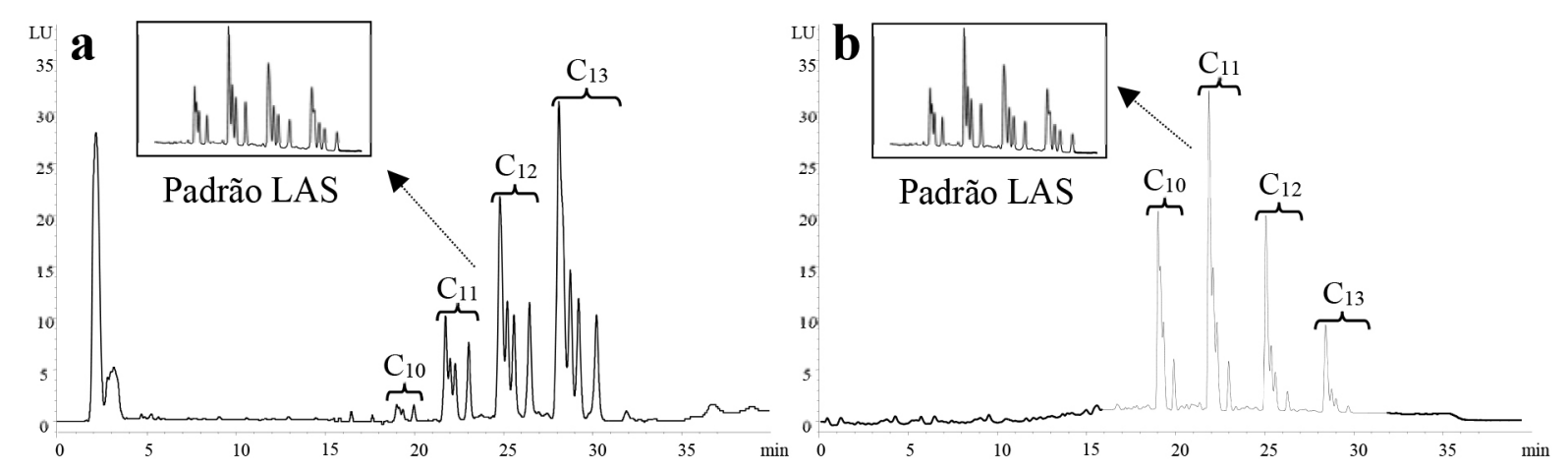

Figura 3. Cromatograma da distribuição dos homólogos do LAS em lodo da ETE e sedimento do rio (a) e afluente da ETE, efluente do reator UASB e água do rio $(b)$ 

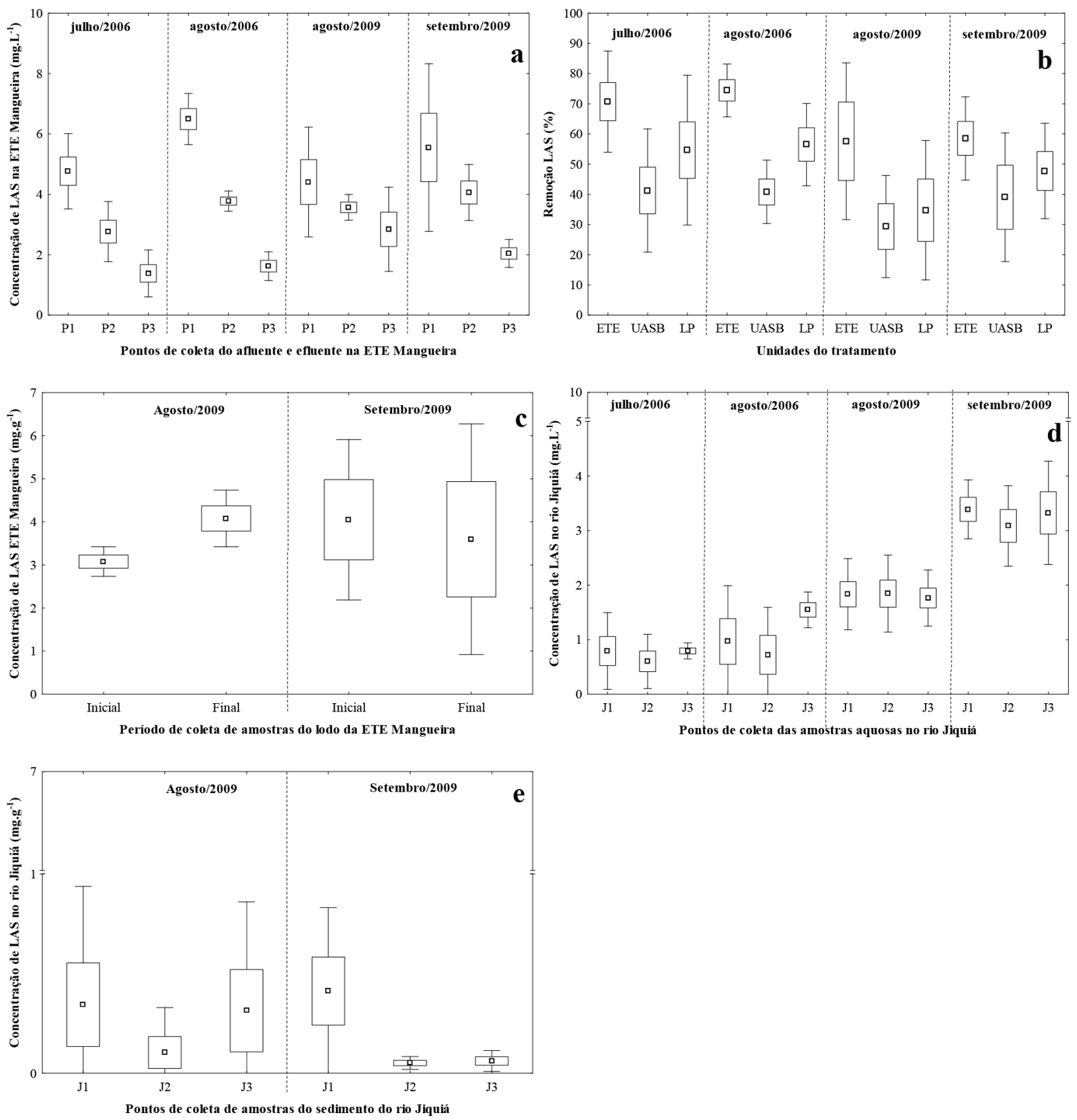

Figura 4. Concentração do LAS na ETE Mangueira em P1 (afluente), P2 (efluente do reator UASB) e P3 (efluente da lagoa de polimento - LP) (a), eficiência de remoção do LAS na ETE, reator UASB e LP (b), concentração do LAS no lodo do reator UASB (c), concentração do LAS no ponto de encontro do efluente da ETE com o rio Jíquiá (J2), em um ponto à montante (J1) e à jusante (J3) do ponto de encontro (d) e no sedimento do rio Jiquiá nos pontos J1, J2 e J3 (e)

para todas as matrizes estudadas. Os surrogates mostraram que as interações do LAS com as diferentes matrizes e colunas de SPE são constantes, o que permitiu utilizá-los também como padrão interno, para corrigir desvios na concentração do LAS, desde a preparação da amostra até a análise cromatográfica.

A ETE Mangueira foi eficiente na remoção do LAS, caracterizando o reator UASB na sua remoção por adsorção ao lodo e a lagoa de polimento por adsorção aos sólidos suspensos e degradação biológica. Nas águas do rio Jiquiá, as concentrações de LAS foram superiores aos valores definidos por órgãos ambientais; e no sedimento também apresentaram valores considerados tóxicos para os organismos do ecossistema terrestre. Ambas as ocorrências são atribuídas ao lançamento de efluentes não tratados.

Os métodos propostos apresentaram a possibilidade de aplicar a coluna $\mathrm{C}_{18}$ para avaliar qualitativamente a degradação do LAS em função de seus isômeros, as diferentes possibilidades de utilização da SPE em relação a diferentes matrizes ambientais; e que o uso do Soxhlet, para extração do LAS de lodo de ETE e sedimento de rio, tem eficiência similar aos métodos de extração ultimamente descritos na literatura, como a extração líquida pressurizada e micro-ondas, estes porém, com maiores custos de aquisição e manutenção.

\section{MATERIAL SUPLEMENTAR}

Algumas tabelas que não foram apresentadas neste texto estão disponíveis em http://quimicanova.sbq.org.br, na forma de arquivo PDF, com acesso livre.

\section{AGRADECIMENTOS}

À DETEN Química (Camaçari - BA) e à CEPSA Química (San Roque, Espanha) pelo apoio a pesquisa sobre LAS; à COMPESA e 

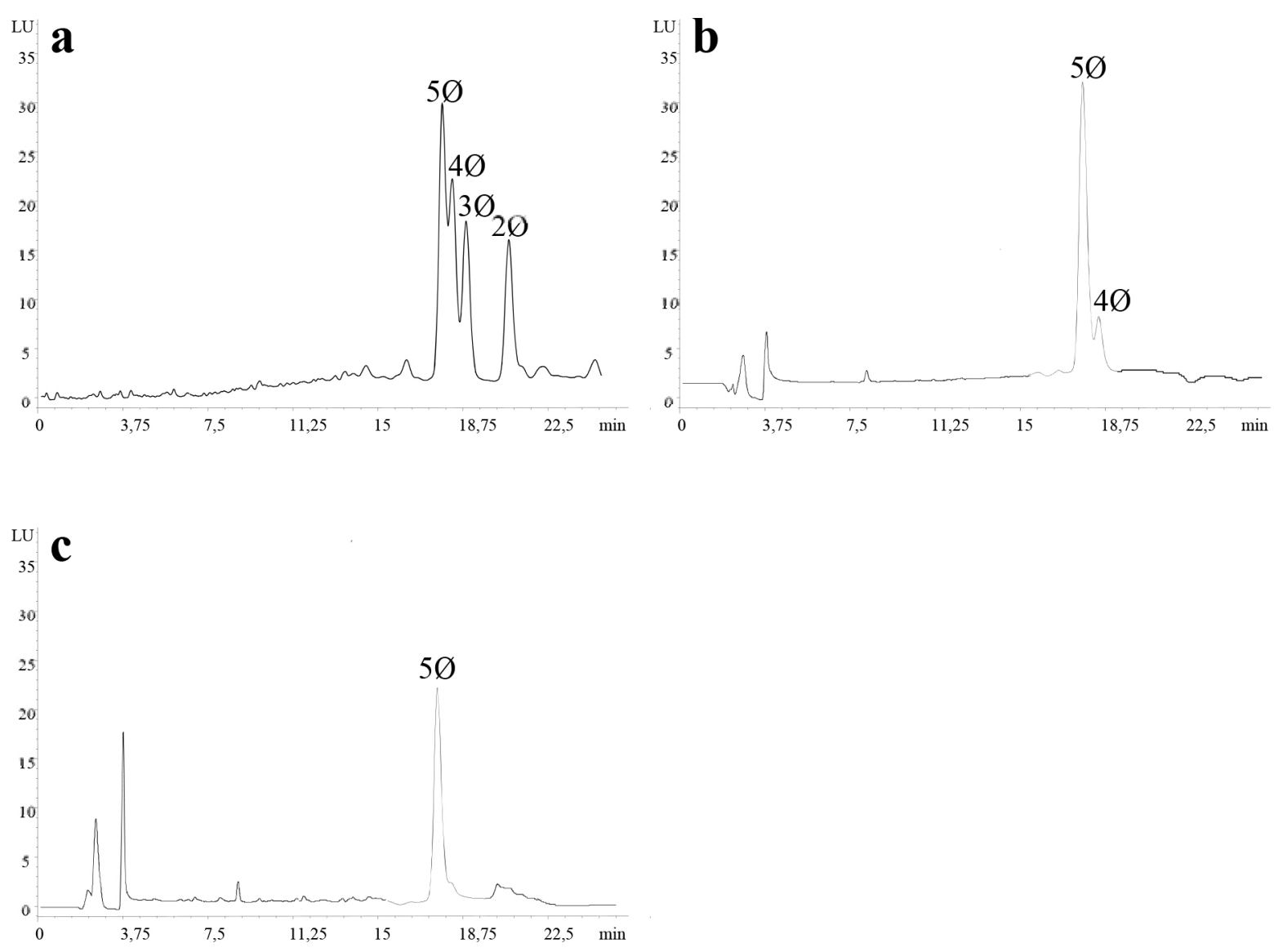

Figura 5. Cromatograma do homólogo $C_{10}$ com os isômeros $2 \emptyset C_{10}, 3 \emptyset C_{10}, 4 \emptyset C_{10}$ e $5 \emptyset C_{10}($ a $)$, com os isômeros $4 \emptyset C_{10}$ e $5 \emptyset C_{10}(b)$ e o isômero $5 \emptyset C_{10}(c)$

à Fibra Revestimentos LTDA pelo apoio aos trabalhos de campo e na estação experimental da UFPE na ETE Mangueira; às agências de fomento Financiadora de Estudos e Projetos (FINEP), Conselho Nacional de Desenvolvimento Científico e Tecnológico (CNPq), Coordenação de Aperfeiçoamento de Pessoal de Nível Superior (CAPES) e Fundação de Amparo à Ciência e Tecnologia do Estado de Pernambuco (FACEPE) pelo auxílio financeiro ao trabalho e pelas bolsas concedidas.

\section{REFERÊNCIAS}

1. Almeida, J. L. G.; Dufaux, M.; Ben Taarit, Y.; Naccache, C.; J. Am. Oil Chem. Soc. 1994, 71, 675.

2. Mungray, A.K.; Kumar, P.; Int. Biodeterior. Biodegrad. 2009, 63, 981.

3. http://www.heraproject.com/files/HERA-LAS\%20revised\%20April\%20 2013\%20Final1.pdf,

acessada em Janeiro 2017.

4. Waters, J.; Holt, M. S.; Mathijs, E.; Tenside, Surfactants, Deterg. 1989, 26, 29.

5. Berna, J. L.; Ferrer, J.; Moreno, A.; Prats, D.; Ruiz, B. F.; Tenside, Surfactants, Deterg. 1989, 26, 101.

6. Wolf, W.; Feijtel, T.; Chemosphere 1989, 36, 1319.

7. Jensen, J.; Sci. Total Environ. 1999, 226, 93.

8. Temmink, H.; Klapwijk, B.; Water Res. 2004, 38, 903.

9. García, M. T.; Campos, E.; Ribosa, I.; Latorre, A.; Sánchez-Leal, J.; Chemosphere 2005, 60, 1636.

10. Cantarero, S.; Prieto, C.A.; López, I.; J. Environ. Manage. 2012, 95 supl., S149.

11. APHA-AWWA-WPCF.; Standard methods for the examination of water and wastewater, $21^{\text {th }}$ ed., APHA: Washington, D.C, 2005.
12. http://www.mma.gov.br/port/conama/res/res05/ res35705.pdf. Acessada em Janeiro de 2017.

13. Waters, J.; Feijtel, T. C. J.; Chemosphere 1995, 30, 1939.

14. Clara, M.; Scharf, S.; Scheffknecht, C.; Gans, O.; Water Res. 2007, 41, 4339.

15. Tabor, C. F.; Barber, L. B.; Environ. Sci. Technol. 1996, 30, 161.

16. Akyuz, M.; Talanta 2007, 71, 471.

17. Moldovan, Z.; Avram, V.; Marincas, O.; Petrov, P.; Ternes, T.; J. Chromatogr. A 2010, 1218, 343.

18. Léon, V.M.; González-Mazo, E.; Gómez-Parra, A.; J. Chromatogr. A 2000, 889, 211

19. Wangkarn, S.; Soisungnoen, P.; Rayanakorn, M.; Grudpan, K.; Talanta 2005, 67, 686.

20. Cantarero, S.; Zafra-Gómez, A.; Ballesteros, O.; Navalón, A.; Vílchez, J. L.; Verge, C.; De Ferrer, J. A.; Environ. Toxicol. Chem. 2011, $30,813$.

21. Alexandre, B.; Barbara, G.; Laure, W.; Bruno, D.; Adriana, G. O.; Emmanuelle, V.; J. Chromatogr. A 2016, 1450, 64.

22. Lara-Martín, P. A.; Gómez-Parra, A.; González-Mazo, E.; J. Chromatogr. A 2006, 1114, 205.

23. Traverso-Soto, J. M.; Lara-Martín, P. A.; González-Mazo, E.; León, V. M.; Sci. Total Environ. 2015, 503-504, 87.

24. Oliver-Rodríguez, B.; Zafra-Gómez, A.; Carmino-Sánchez, F. J.; Conde-González, J. E.; Pérez-Trujillo, J. P.; Vílchez, J. L.; Microchem. J. 2013, 110,158 .

25. Oliver-Rodríguez, B.; Zafra-Gómez, A.; Reis, M. S.; Duarte, B. P. M.; Verge, C.; De Ferrer, J. A.; Pérez-Pascual, M.; Vílchez, J. L.; Chemosphere 2015, 131, 1.

26. http://www.cprh.pe.gov.br/Publicacoes/Rimas/2009/41788\%3B70757\% 3B490201\%3B0\%3B0.asp, Acessada em Janeiro 2017.

27. Pimentel, M. F.; Barros-Neto, B.; Quim. Nova 1996, 19, 268. 
28. Ribani, M.; Bottoli, C. B. G.; Collins, C. H.; Jardim, I. C. S. F.; Melo, L. F. C.; Quim. Nova 2004, 27, 771.

29. Economou, A.; Botitsi, H.; Antoniou, S.; Tsipi, D.; J. Chromatogr. A 2009, 1216, 5856 .

30. Cerqueira, M. B. R.; Dias, A. N.; Caldas, D. S. S.; Santana, F. B.; D’Oca, M. G. M.; Primel, E. G.; Quim. Nova. 2011, 34, 156.

31. Instituto Nacional de Metrologia, Normalização e Qualidade Industrial (INMETRO); Orientações sobre Validação de Métodos de Ensaios Químicos, DOQ-CGCRE-008, 2003.

32. Agência Nacional de Vigilância Sanitária (ANVISA); Resolução RE $n^{\circ}$ 899, de 29/05/2003.
33. Lara-Martin, P. A.; Gómez-Parra, A.; González-Mazo, E.; J. Chromatogr. A 2006, 1114, 205.

34. Lara-Martin, P. A.; González-Mazo, E.; Brownawell, B. J.; J. Chromatogr. A 2011, 1218, 4799.

35. Dong, W.; Eichhorn, P.; Radajewski, S.; Schleheck, D.; Denger, K.; Knepper, T. P.; Murrell, J. C.; Cook, A. M.; J. Appl. Microbiol. 2004, 96, 630.

36. Schleheck, D.; Knepper, T. P.; Eichhorn, P.; Cook, A. M.; Appl. Environ. Microbiol. 2007, 73, 4725. 